and

$$
v_{i}(x)=\sum_{k=1}^{i} B_{i k} w_{k}(x) .
$$

Since $B_{i k} \leqq\left\{\sum_{j=1}^{\infty} B_{j k}^{2}\right\}^{1 / 2}=l_{i-1, k}$, in all the cases of the ratio function $r(x)$ considered, the right-hand members of (4) and (5) are absolutely convergent and bounded, wherever, respectively, the $v$ 's and $w$ 's are bounded. Hence, if conditions are such that the right-hand member of (4) converges to the value of the left-hand member and if a set of points is known for which the v's are bounded, then the w's are bounded on the same set except where $r(x)=0$. Similarly, boundedness of the w's leads through (5) to results on the boundedness of the $v$ 's.

UnIVERSITY OF Minnesota

\title{
A MAPPING CHARACTERIZATION OF PEANO SPACES
}

O. G. HARROLD, JR.

The Hahn-Mazurkiewicz theorem states that any Peano space (compact, connected, locally connected, metric space) is a continuous image of the interval $0 \leqq t \leqq 1$, and conversely. Clearly, the mapping function is not uniquely determined. If the Peano space $\mathscr{X}$ has special topological properties, the mapping may be selected in a simpler fashion than might be expected generally. On the other hand, special properties of $\mathcal{X}$ may impose certain necessary restrictions on the mapping. For example, if $\mathcal{X}$ is a regular continuum in the sense of Menger, then, by a theorem due to Nöbeling, ${ }^{1}$ there is a continuous mapping $f$ of the circle ${ }^{2}$ onto $\mathcal{X}$ such that each point of finite order is covered by the mapping a number of times which does not exceed the order of the point. That is, if $o(x)$ is the order of the point $x$ and $m(x)$ is the number of points in $f^{-1}(x)$, then $m(x) \leqq o(x)$ for each point for which $o(x)$ is finite. On the other hand, if $\mathcal{X}$ is of dimension $n$, then any continuous mapping of a 1-dimensional compact set onto $\mathscr{X}$,

\footnotetext{
${ }^{1}$ G. Nöbeling, Reguläre Kurven als Bilder der Kreislinie, Fundamenta Mathematicae, vol. 20 (1933), pp. 30-46.

2 The interval may be used instead of the circle if we make $f(0)=f(1)$ and count inverses on $0 \leqq t<1$.
}

Presented to the Society, September 5, 1941; received by the editors October 21, 1941. 
in particular, of an interval or circle, is such that there is a dense set of points in the $n$-dimensional part of $\mathcal{X}$ each of which has at least $n$ inverse points in the original set. ${ }^{3}$

Denote the set of local separating points of the Peano space $\mathcal{X}$ by $\mathcal{L}:{ }^{4}$ If $\mathscr{x} \subset \overline{\mathscr{X}-\mathcal{L}}$, that is, if $\mathscr{X}$ contains no free arcs, ${ }^{5}$ there is a strongly irreducible mapping of the interval $J$ or circle $\mathcal{C}$ onto $\mathcal{X} .^{6}$ That is, for such spaces there exist continuous mappings of $J$ or $\mathcal{C}$ onto $\mathcal{X}$ such that no proper closed subset maps onto the whole space. Thus if $\mathcal{X}$ is an $n$-dimensional sphere and $f$ is a strongly irreducible mapping of $J$ onto $\mathcal{X}$, there is a dense set of points each covered at least $n+1$ times and also a dense set of points each covered just once.

In addition to the symbols $f, \mathscr{X}, \mathcal{L}, m(x)$ and $o(x)$ used above, the following notations will be observed. Let $\psi$ denote the aggregate of points $x \in \mathcal{X}$ lying in an open free arc of $\mathcal{X}-a-b$. If for a (continuous) mapping of $\mathfrak{I}$ into a subset of $\mathcal{X}, y \in \psi$ implies $m(y) \leqq 2$, the mapping will be said to be of type $\mathfrak{M}$.

Theorem 1. Let $a$ and $b$ be points of the Peano space $X$. There is a continuous mapping of the interval $0 \leqq t \leqq 1$ onto $\mathcal{X}$ of type $\mathfrak{M}$ such that $f(0)=a, f(1)=b$.

The theorem asserts, essentially, that there is a mapping of $J$ onto $X$ such that every free arc is swept through at most twice.

The following lemmas will be useful in the proof of Theorem 1.

Lemma 1.1. If $D$ is a subcontinuum of the dendrite $D^{0}$, to $e>0$ there is a finite collection $D^{1}, D^{2}, \cdots, D^{n}$ of dendrites in $D^{0}$ such that $D=D^{1} \subset D^{2} \subset \cdots \subset D^{n}=D^{0}$ and each component of $D^{i+1}-D^{i}$ has a diameter less than or equal to $e$.

Let $\rho$ be a convex metric ${ }^{7}$ on $D^{0}$. Let $d=$ glb of numbers $r$ such that

${ }^{3}$ If the original set is locally euclidean, the phrase at least $n$ may be replaced by at least $n+1$. See $\mathrm{W}$. Hurewicz, Über dimensionserhohende stetige Abbildungen, Journal für die reine und angewandte Mathematik, vol. 169 (1933), pp. 71-78.

${ }^{4}$ The point $p$ is called a local separating point of $M$ provided that to every neighborhood $U$ of $p$ there is some pair of points of the component of $U$ containing $p$ which is separated in $U-p$.

${ }^{5}$ The set $A$ is called a free $\operatorname{arc}$ of $M$ provided $A$ is an $\operatorname{arc}$ and the interior of $A$ is open in $M$. An open free arc is an open subset of $M$ which is homeomorphic to $0<x<1$. A point is said to lie in an open free arc provided there is a neighborhood of the point in $M$ which is an open free arc. It is to be noted that if $M$ is an arc, neither end point lies in an open free arc.

${ }^{6} \mathrm{O}$. G. Harrold, Jr., A note on strongly irreducible maps of an interval, Duke Mathematical Journal, vol. 6 (1940), pp. 750-752.

7 The metric $\rho$ is called convex after Menger provided that to each pair of distinct points $x$ and $y$ in $M$ there is a point of $M-x-y$ such that $\rho(x, z)+\rho(z, y)=\rho(x, y)$. 
$D^{0} \subset S(D, r)$. Each of the sets $D^{i}=S[D,(i-1) e], i=1,2, \cdots, n$ is a subcontinuum of $D^{0}$. The sets $D^{i}$ satisfy our requirements, where $n$ is the smallest in teger such that $(n-1) \geqq d$.

Lemma 1.2. Let $\mathcal{X}$ be a Peano space. There is a sequence $\left(T_{i}\right)$ of dendritic graphs in $\chi$ such that (a) $\lim T_{i}=\mathcal{X}$, (b) $T_{i+1} \supset T_{i}$, and (c) each component of $T_{i+1}-T_{i}$ has a diameter less than or equal to $1 / 2^{i+1}$.

That a sequence of dendrites exists in $\mathcal{X}$ satisfying (a) and (b) is well known. Application of Lemma 1.1 to the successive terms of this sequence gives the desired result.

Proof of Theorem 1. The theorem is true for a connected dendritic graph with $n$ end points, as a simple induction shows. Suppose, temporarily, that neither $a$ nor $b$ lies in an open free arc. Let $T_{1}$ be a dendritic graph in $\mathcal{X}$ containing $a$ and $b:^{8}$ Let $f_{1}$ denote a continuous mapping of type $\mathfrak{M}$ of $\mathfrak{J}$ onto $T_{1}$ with $f_{1}(0)=a, f_{1}(1)=b$. Let $\left(T_{i}\right)$ be a sequence of dendritic graphs satisfying Lemma 1.2. Since $T_{2}$ is a graph, $T_{2}-T_{1}$ has but a finite number of components which may be denoted by $C_{1}^{1}, C_{2}^{1}, \cdots, C_{n_{1}}^{1}$. Each $\bar{C}_{i}^{1} \cdot T_{1}$ is a point $c_{i}$. Let $x_{i} \in f_{1}^{-1}\left(c_{i}\right)$. By a rearrangement of notation it may be supposed that $0 \leqq x_{1}<x_{2}$ $<\cdots<x_{p_{1}} \leqq 1, p_{1} \leqq n_{1}$, where each $x_{i}$ corresponds to a distinct $c_{i}$. Set $d_{1}=\min \left|x_{i}-x_{j}\right|, i \neq j,\left|x_{i}\right|, x_{i} \neq 0,\left|1-x_{i}\right|, x_{i} \neq 1$. To $\epsilon=1 / 2$ there is a $d_{2}>0$ such that $|x-y|<d_{2}$ implies $\rho\left[f_{1}(x), f_{1}(y)\right]<\epsilon / 2=1 / 2^{2}$, where $\rho$ denotes the metric of $\mathcal{X}$. Put $W=S\left(x_{1}+x_{2}+\cdots+x_{p_{1}}, d / 3\right)$, where $d=\min \left(d_{1}, d_{2}\right)$. Let $J_{i}$ be the component of $W$ containing $x_{i}$. Let $I_{1}, I_{2}, \cdots, I_{p_{1}+1}$ be the intervals on $J$ complementary to $W$, where $I_{1}$ becomes degenerate if $x_{1}=0$ and $I_{p_{1}+1}$ degenerate if $x_{p_{1}}=1$. The interval $J$ is now subdivided into the intervals (in order) $I_{1}, \bar{J}_{1}, I_{2}, \bar{J}_{2}, \cdots, I_{p_{1}+1}$. Let $t$ denote the piecewise linear map obtained by sending $I_{1}$ onto $\left(0 x_{1}\right)$ with order preserved, $I_{2}$ onto $\left(x_{1} x_{2}\right), \cdots, I_{p_{1}+1}$ onto $\left(x_{p_{1}} 1\right)$. For $x \in \sum I_{i}$, put $f_{2}(x)=f_{1}[t(x)]$. On $\bar{J}_{i}$ define a map $g_{i}$ of the desired type so that $g_{i}\left(\bar{J}_{i}\right)=D_{i}$. where $D_{i}$ is the enclosure of all components $C_{i}^{1}$ having $c_{i}$ as a limit point. The set $D_{i}$ is a dendrite of diameter less than or equal to $1 / 2^{2}$. The map $g_{i}$ may be so selected that for the end points of $J_{i}, g_{i}=f_{2}$.

See K. Menger, Untersuchungen über allgemeine Metrik, Mathematische Annalen, vol. 100 (1928), pp. $81 \mathrm{ff}$. For the existence of the metric assumed here, see C. Kuratowski and Whyburn, Sur les elements cycliques et leurs applications, Fundamenta Mathematicae, vol. 16 (1930), pp. 305-331.

${ }^{8} T_{1}$ could be taken to be an arc but in order that the discussion to follow be general it is assumed only to be a connected linear graph containing no simple closed curve. 
The definition of $f_{2}$ is now completed: if $x \in \bar{J}_{i}, f_{2}(x)=g_{i}(x)$. Clearly, $f_{2}(J)=T_{2}$ and $f_{2}$ is continuous. For $x \in \sum I_{i},|x-t(x)|<d$, hence $\rho\left[f_{1}(x), f_{2}(x)\right]<1 / 2^{2}$. If $x \in \bar{J}_{i}, \rho\left[f_{1}(x), f_{1}\left(x_{i}\right)\right]<1 / 2^{2}$, and since the diameter of $D_{i}$ is less than or equal to $1 / 2^{2}, \rho\left[f_{2}(x), f_{2}\left(t^{-1}\left(x_{i}\right)\right)\right]<1 / 2^{2}$. But $f_{2}\left(t^{-1}\left(x_{i}\right)\right)=f_{1}\left(x_{i}\right)$, hence by the triangle inequality $\rho\left[f_{1}(x), f_{2}(x)\right]$ $<1 / 2$. Thus if $\sigma$ denotes the usual metric of the function space $X^{\top}$, $\sigma\left(f_{1}, f_{2}\right) \leqq 1 / 2 .{ }^{9}$

To show that $f_{2}$ is of type $\mathfrak{M}$ consider a point $y \in \psi \cdot T_{2}$. It is to be shown that $f_{2}^{-1}(y)$ contains at most two points, that is, $m\left(y, f_{2}\right) \leqq 2$. If $y \in T_{2}-T_{1}, y$ lies in an unique $D_{i}$, hence $m\left(y, f_{2}\right) \leqq 2$. If $y \in T_{1}-\sum D_{i}$, $f_{2}^{-1}(y)=t^{-1} f_{1}^{-1}(y)$. Since $f_{1}$ has the desired property and $t$ is $1-1$ on the set composed of the interiors of the intervals $I_{i}, m\left(y, f_{2}\right) \leqq 2$. Consider the remaining case $y=c_{i}$. Here $m\left(y, f_{1}\right)=1$, for suppose, on the contrary, $f_{1}^{-1}(y) \supset q_{1}+q_{2}$. Since $y \in \psi, y \neq a, b$, hence $q_{1}$ and $q_{2}$ divide $J$ into three subintervals $A, B$ and $C$. But each of these subintervals has as an image under $f_{1}$ a nondegenerate continuum containing $y$. Hence points of $T_{1}$ near $y$ have three inverses on 3 , which denies the property of $f_{1}$. Since $y \in \psi$, only one component $C_{i}^{1 \prime}=D_{i}$ of $T_{2}-T_{1}$ can have $y$ as a limit point. The mapping $g_{i}$ has the desired property, thus $f_{2}^{-1}(y)=g_{i}^{-1}(y)+f_{1}^{-1}\left[t^{-1}(y)\right]$ is precisely a pair of points. Hence for $y \in \psi \cdot T_{2}, m\left(y, f_{2}\right) \leqq 2$, and $f_{2}$ is of type $\mathfrak{M}$.

The general inductive hypothesis is now clear.

To the dendrite $T_{n}$ there is a continuous mapping $f_{n}, f_{n}(\mathfrak{J})=T_{n}$, of type $\mathfrak{M}$ and such that $f_{n}(0)=a, f_{n}(1)=b$. Further, $\sigma\left(f_{i-1}, f_{i}\right) \leqq 1 / 2^{i-1}$, $i=2,3, \cdots, n$. The construction of $f_{n+1}$ from $f_{n}$ is accomplished precisely as above.

There is thus determined a sequence of points $\left(f_{n}\right)$ of the space $X^{J}$ such that to $e>0$ there is an index $N$ such that for $i, j>N, \sigma\left(f_{i}, f_{j}\right)<e$. The space $\chi^{\mathfrak{J}}$ being complete, let $\lim f_{n}=f$. Clearly, $f(\mathfrak{J})=\chi$. To complete the proof of Theorem 1 it will suffice to show that $y \in \psi$ implies $f^{-1}(y)$ has at most two components. For, if we grant this, the factorization $f=k[h(x)]$, where $h$ is the monotone transformation obtained by shrinking the components of $f^{-1}(y)$ into points and $k$ is the corresponding light transformation, yields $k(\mathfrak{J})=\mathcal{X}, m(y \in \psi, k) \leqq 2$, hence $k$ is of type $\mathfrak{M} .^{10}$

Suppose, on the contrary, $y \in \psi$ and $X_{1}, X_{2}$ and $X_{3}$ are three components of $f^{-1}(y)$. Since $y \in \psi, y \neq a, b$, hence $J-\sum X_{i}$ has precisely 4 components $R_{i}, i=1,2,3$ and 4 . Suppose $w_{i}$ and $t_{i}$ are the left and right end points of $X_{i}$, respectively. (If $X_{i}$ is a point, $w_{i}=t_{i}$.) Let the

${ }^{9}$ If $f, g \in X \mathcal{J}, \sigma(f, g)=\operatorname{lub} \rho[f(x), g(x)], x \in J$.

${ }^{10}$ This is an application of a factor theorem for continuous transformations due to Eilenberg and Whyburn. 
notation be arranged so that $w_{i}=\bar{R}_{i} \cdot X_{i}, i=1,2$ and 3 . The points $w_{i}$ and $w_{j}, i \neq j$, are, of course, distinct. Let $A_{i}, i=1,2$ and 3 be a subinterval of $\bar{R}_{i}$ containing $w_{i}$ such that $f\left(A_{i}\right) \subset U$, where $U$ is any fixed open free arc of $\chi$ containing $y$. Let $B_{i+1} i=1,2$ and 3 be a proper subinterval of $R_{i+1}-A_{i+1}$ containing $t_{i}$ such that $f\left(B_{i}\right) \subset U$, where $A_{4}=0$ by definition. Since $X_{i}$ is a component of $f^{-1}(y)$, the sets of $f\left(A_{i}\right), f\left(B_{i}\right)$ are nondegenerate subarcs of $U$ with at least the point $y$ common. Some point $y^{0}$ of $U-y$ must be covered by at least three of these six sets. Denote three of the corresponding sets $A_{i}\left(B_{i}\right), i=1,2$ and 3 by $C_{1}, C_{2}$ and $C_{3}$. One end point of $C_{i}$, say $a_{i}$, maps into $y$. As $C_{i}$ is traversed from $a_{i}$ let $b_{i}$ be the first point in $f^{-1}\left(y^{0}\right)$. Let $G$ denote any subarc of $U-y-y^{0}$ which lies between $y$ and $y^{0}$. Set $d=\rho\left(G, y+y^{0}\right)$ $>0$. Then for $n$ large enough $\sigma\left(f_{n}, f\right)<d / 3$. But $f_{n}\left(a_{i} b_{i}\right)$ is a connected subset of $U$ which contains a point from each component of $U-G$, hence $f_{n}\left(a_{i} b_{i}\right) \supset G$. This denies that $f_{n}$ is of type $\mathfrak{M}$. The proof of Theorem 1 under the special restriction that neither $a$ nor $b$ lies in a free arc has been completed.

To remove the restriction suppose first that only $a$ lies in a free arc of $\mathcal{X}$. Imagine that $\mathcal{X}$ is situated in the Hilbert cube and let $\mathcal{A}$ be an arc which is joined onto $\mathcal{X}$ at $a$ and has no other point in $\mathcal{X}$. Let $a^{1}$ be the other end point of $\mathcal{A}$. Construct a mapping as above with $f(0)=a^{1}, f(1)=b, f(J)=\chi+\mathcal{A}$. Since neither $a^{1}$ nor $b$ lies in an open free arc of $X+\mathcal{A}$, such a mapping will exist. Let $x^{1}$ be the least $x$ for which $f(x)=a$. Then the mapping $f$ on the interval $x^{1} \leqq t \leqq 1$ satisfies our requirements. A similar modification suffices to treat the case in which $b$ is an open free arc and also the case in which both $a$ and $b$ have this property.

Set $\mathscr{W}=X-\bar{\psi}$. The set $\mathscr{W}$ is open. Put $\mathscr{Q}=\psi+(\mathscr{W} \cdot \mathcal{N})$, where $\mathcal{N}$ is the set of nonlocal separating points of $\mathcal{X}$. We come now to the principal result.

TheOREM 2. Let $a$ and $b$ be points of the metric space $\mathcal{X}$. In order that $\chi$ be a Peano space it is necessary and sufficient that for any countable subset $\mathcal{P}$ of $2-a-b$ there be a continuous mapping $f$ of $0 \leqq t \leqq 1$ onto $\mathcal{X}$ such that $f(0)=a, f(1)=b$ and $y \in \mathcal{P}$ implies $m(y) \leqq 2$.

Proof. The sufficiency is clear. If $\psi=0$, the result is known, in fact, in this case a mapping of the described type exists such that for $y \in \mathcal{P}$, $m(y)=1 .^{6}$ It is supposed, then, that $\psi \neq 0$. By application of Theorem 1 , there is a mapping of type $\mathfrak{M}$ of $\mathfrak{J}$ onto $\mathcal{X}$ with $f(0)=a, f(1)=b$. The desired map will be obtained by a modification of $f$.

To facilitate the discussion it will be supposed that $\chi$ has an $S$-metric, that is, a metric $\rho$ such that for each $r>0$ and $x \in \mathcal{X}, \bar{S}(x, r)$ is a lo- 
cally connected continuum. ${ }^{11}$ Let $A=a_{1}+a_{2}+\cdots=\mathscr{P} \cdot \mathscr{W}=\mathbb{P} \cdot W \cdot$. . Set $d_{1}=\rho\left(a_{1}, X-W\right)$. Choose a number $e_{1}$ such that $d_{1} / 2<e_{1}<d_{1}$ and $A \cdot\left\{\bar{S}\left(a_{1}, e_{1}\right)-S\left(a_{1}, e_{1}\right)\right\}=0$. This is possible since $A$ is countable. Let $a_{k_{2}}$ be the first point of $A$ in $\mathscr{W}-\bar{S}_{1}$, where $S_{1}=S\left(a_{1}, e_{1}\right)$. Let $d_{2}=\rho\left[a_{k_{2}},(X-\mathscr{W})+S_{1}\right]$. The number $e_{2}$ is chosen so that $d_{2} / 2<e_{2}<d_{2}$ and $A \cdot\left\{\bar{S}\left(a_{k_{2}}, e_{2}\right)-S\left(a_{k_{2}}, e_{2}\right)\right\}=0$. Continuing in this way a sequence of spheres $\left(S_{i}\right)$ is determined such that (a) $\bar{S}_{i}$ is a Peano space, (b) $\bar{S}_{i} \cdot \bar{S}_{j}=0, i \neq j$, (c) $\delta\left(S_{i}\right) \rightarrow 0$, (d) $\sum \bar{S}_{i}=\overline{\mathscr{W}}$ and (e) $A \cdot\left(\bar{S}_{i}-S_{i}\right)=0$.

Set $V_{i}=f^{-1}\left(S_{i}\right)$. Let $V_{i j}, j=1,2, \cdots$ be the components of $V_{i}$. Let $V_{i 1}$ be a component of $V_{i}$ such that $V_{i 1} \cdot f^{-1}\left(a_{k_{i}}\right) \neq 0$. Every point of $\bar{S}_{i}$ is either a nonlocal separating point of $\bar{S}_{i}$ or a limit point of such points. This is clear if $x \in S_{i}$, for $S_{i} \subset \mathcal{W}$. If $x \in \bar{S}_{i}-S_{i}, x$ is a limit point of points of $S_{i}$ and hence a limit point of nonlocal separating points of $\bar{S}_{i}$. Thus, having shown that $\bar{S}_{i}$ is a Peano space with no free arcs, there is a strongly irreducible mapping, $f_{i 1}\left(V_{i 1}\right)=\bar{S}_{i}$, such that $f=f_{i 1}$ on $\bar{V}_{i 1}-V_{i 1}$ and $y \in \mathcal{P} \cdot \bar{S}_{i}$ implies $f_{i 1}^{-1}(y)$ is a single point.

On $V_{i j}, j>1$, two cases are distinguished according as $f$ maps the end points of $V_{i j}$ into the same point or not. If $f$ carries the end points of $V_{i j}$ into $x$, define $f_{i j} \equiv x$ on $\bar{V}_{i j}$. If $f$ carries the end points of $V_{i j}$ into distinct points $x$ and $y$, proceed as follows. The set $\bar{S}_{i}-A$ is a connected and locally connected $G_{\delta}$ set $^{12}$ in a complete space, hence there is an arc $R_{i j} \subset \bar{S}_{i}-A$ which joins $x$ and $y .{ }^{13}$ On $\bar{V}_{i j}$ define $f_{i j}$ to be a homeomorphism into $R_{i j}$ such that $f_{i j}$ agrees with $f$ on $\bar{V}_{i j}-V_{i j}$.

The new mapping $g$ will now be defined. On $J-\sum V_{i j}$, set $g(x) \equiv f(x)$. On $V_{i j}$, set $g(x)=f_{i j}(x)$. Since $f$ agrees with $g$ on the end points of $V_{i j}$ and each $f_{i j}$ is continuous, $g$ is continuous (we use here the condition (c) on the spheres $\left.\left(S_{i}\right)\right)$. Clearly, $g(\mathfrak{J})=$ X. If $y \in \mathcal{P} \cdot \psi, m(y, f)$ $=m(y, g) \leqq 2$, by virtue of the fact that $f$ is of type $\mathfrak{M}$. If $y \in \mathcal{P} . \mathscr{W}$, $y$ lies in an unique $S_{i}$ and $g^{-1}(y)=f_{i 1}^{-1}(y)$, hence $m(y, g)=1$.

\section{NORTHWESTERN UNIVERSITY}

${ }^{11} \mathrm{~J}$. L. Kelley, A metric connected with property $S$, American Journal of Mathematics, vol. 61 (1939), pp. 764-768.

${ }^{12}$ The complement of a countable set of nonlocal separating points in a Peano space is connected and locally connected, see G. T. Whyburn, Semi-closed sets and collections, Duke Mathematical Journal, vol. 2 (1936), pp. 685-690.

${ }^{13}$ This is the well known Moore-Menger generalization of the arcwise connectivity theorem for regions in a Peano space. 